\title{
Editorial
}

\section{Special issue on modeling and control technologies for civil, energy, and power systems}

Energy and power systems technology is a fundamental field in the civil engineering. Innovation in power generation, grid intelligence, renewable resources, propulsion and powertrains for transportation devices, etc. is a long-term challenging theme for science and technology. Especially, improving the efficiency and the emission in energy consumption attracted very much attention in this technology developing field, and the attention is recently focused on the control technologies. In fact, whether the targeted system is a simple device such as electrical motor or a complex large-scale system such power grid, to deal with it as a dynamical system and to provide real-time control strategy decision based on the model that describes the system behavior, are basic stance and in general the real-time control aims the performance improvement involving the accuracy of manufactory, the efficiency optimality etc.

This special issue presents a collection of 9 papers that provide a demonstration of recent innovative researches in modeling and control of energy and power systems. The papers are divided into three topics: electric power systems, combustion engines, and electric devices. For the first topic, Y. Okawa and T. Namerikawa proposed a real-time control approach to demand-supply balancing and voltage phase difference rejection problem. W. Wang and $\mathrm{H}$. Ohmori addressed load frequency control problem for two-area interconnected power system with a new LMI-based algorithm, and a nonlinear control method adapted to plug-in device of power grid is investigated by A. Tilli and C. Conficoni. Concerning the second topic, two challenges of advanced control of internal combustion engine are collected provided by C. Khajorntraidet and K. Ito, M. Kumar and T. Shen that show the potentiality in developing advanced control of gasoline engines with cylinder pressure sensor. The last block involves several control technologies of devices. O. Bagherieh and R. Nagamune proposed a gain-scheduling control for wind turbine, K. Z. Liu et al. presented a new adaptive control strategy for induction motor. M. A. Rahman et al. investigated piezoelectric micro-actuator control for high precision dual-stage systems, and M. Dueck et al. presented a challenge in active turbulent flow control with developed rea-time actuator and sensor network.

Certainly, to innovate the energy and power technology with modeling and control in civil engineering is still an open field with a lot of potentialities. We hope that this special issue of Control Theory and Technology will encourage researchers to challenge these modeling and the control technologies. We would like to acknowledge the Editr-in-Chief, Professor Yiguang Hong, and his staff for the strong support through the editing process.

Guest editors:

Tielong Shen

Sophia University, Tokyo, Japan

Kang-Zhi Liu

Chiba University, Chiba, Japan

Shunshoku Kanae

Fukui University of Technology, Fukui, Japan

(c) 2015 South China University of Technology, Academy of Mathematics and Systems Science, CAS, and Springer-Verlag Berlin Heidelberg 